\title{
Asymmetrical Sensing Configuration for Improved Sensitivity in Calorimetric High Flow Measurements in Constant Power Mode
}

\author{
Vasileios Kitsos, Matthew Schormans, Andreas Demosthenous and Xiao Liu \\ Department of Electronic \& Electrical Engineering, University College London, WC1E 7JE, UK \\ Email: \{v.kitsos; matthew.schormans.10; a.demosthenous; xiao\}@ucl.ac.uk
}

\begin{abstract}
This work compares the resolution of calorimetric flow sensors in constant power mode using symmetrically located sensing elements to those using asymmetrical locations, for flow rates higher than the turn-over point. The experimental results show that the resolution can be improved up to six times by placing the temperature sensors in unequal distances, for the same power consumption.
\end{abstract}

\section{INTRODUCTION}

Flow sensors play an important role in various fields such as industrial processes, environmental monitoring [1] and in medical diagnostics [2][3]. The principle of a flow measurement can be based on a variety of topologies and techniques and it can be divided into two main categories: thermal [4][5] and nonthermal flow sensors [6][7][8]. The latter are usually complex and their fabrication can be expensive. In contrast, thermal flow sensors rely on heat transfer phenomena to determine a fluid's velocity and have no moving parts, hence, they potentially offer higher reliability and are less expensive to make. It is also possible to implement thermal type flow sensors in a standard CMOS process, making it easier to be integrated with the rest of the circuitry.

Calorimetric is one of the most popular thermal flow sensor topologies due to the flow direction information and its quicker response time. It consists of a central heating element and two equally spaced temperature sensors, one at the upstream and the other one at the downstream of the flow. Its operation is based on the flow-induced temperature gradient. When flow occurs, the originally symmetrical heat distribution is disturbed and a thermal gradient occurs where temperature is low at the upstream and high at the downstream. The temperature difference between the two temperature sensors placed at the inlet and outlet can be translated to the flow's velocity [9]. In addition, calorimetric flow sensors can operate in different modes, depending on the conditions the power is delivered to the heater. It has been proven that when they operate in constant power $(\mathrm{CP})$, the temperature difference $\Delta T$ between two symmetrically positioned temperature sensors increases almost linearly for low velocities, then it reaches a maximum often called turn-over velocity, and finally, it decreases with increasing flow rate [10][11].

The effect of distance between the heater and the sensing

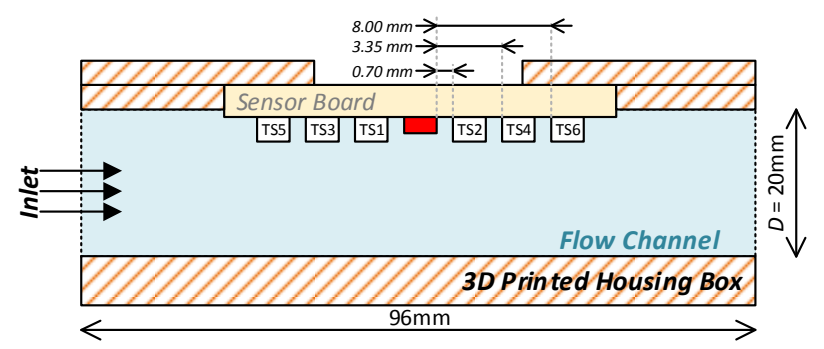

Fig. 1: Cross-sectional drawing view of the housing where sensor board is placed. The heating element is in red color. TS1 to TS6 are the temperature sensors 1 to 6 .

elements of a symmetrical pair has been studied quite extensively [12][13]. However, this is not the case for asymmetrical locations of temperature sensors. Nguyen and Dotzel [11] firstly used asymmetric topologies for flow measurements using $\mathrm{CP}$ mode for low flow rates $(\mu \mathrm{L} / \mathrm{min})$. However, they preferred to use one pair of temperature sensing elements and multiple asymmetrically located heating elements. Such a topology has the benefit of smaller transducer area when thermopiles are used, however, the flow sensor can operate using only symmetrical or asymmetrical locations at a time, but not combined. In [14], the authors have also studied the asymmetrical position of temperature sensing elements for constant current (CI) and constant temperature (CT) mode. However, as the authors stated, the CI measurements on a heater of high temperature coefficient are not as reliable as CT, while CT mode is not easy to implement.

On the other hand, CP offers simple implementation and reproducible measurements. This work investigates and proves that the use of asymmetrical locations in CP mode improves substantially the sensitivity of the flow sensor after the turn-over velocity, a range sometimes unused due to its low performance in this mode. In the following sections, the experimental setup and results are presented.

\section{SYSTEM SETUP}

\section{A. Sensor Board and Housing Box}

Fig. 2 depicts the calorimetric flow sensor used for the experiments and it is made of off-the-shelf components mounted on a typical $1.6 \mathrm{~mm}$ thick FR4 printed circuit board (PCB). It 


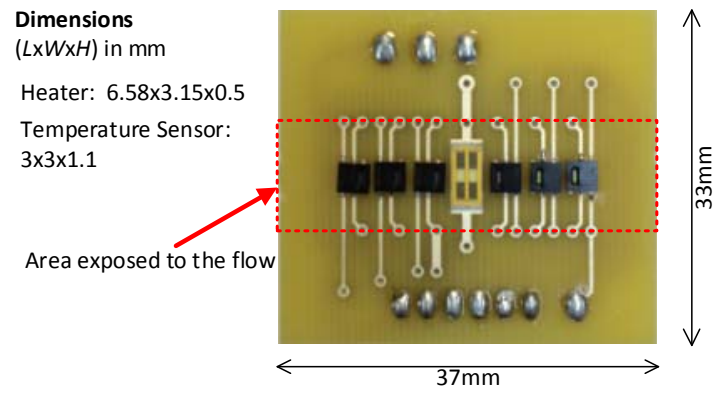

Fig. 2: $\quad$ Photo of the sensor board. The dimensions are stated and the area exposed to fluid is demonstrated.

consists of a surface mount $10 \Omega$ thin film resistor from Vishay ${ }^{\circledR}$ used as a heater, and six digital IC temperature sensors from Sensirion $^{\circledR}$ (STS21). PCB offers a relatively low thermal conductivity compared to Si-based flow sensors, hence, MEMs techniques are not needed in order to achieve thermal isolation of the sensor's components. The temperature coefficient of the heater is $25 \mathrm{ppm}$ which corresponds to a maximum deviation of resistance of $0.125 \%$ for a temperature range of $50{ }^{\circ} \mathrm{C}$. In such a case, constant current on the heater can be considered as constant power with negligible error. The maximum power rating of the resistor is $2.5 \mathrm{~W}$. The digital IC temperature sensors are placed in pairs symmetrically in respect to the heater and in distances of $0.7,3.35$ and $8 \mathrm{~mm}$. Each of these sensors incorporates a 14-bit ADC that provides a resolution of $0.01{ }^{\circ} \mathrm{C}$ and they can communicate with a micro-controller $(\mu \mathrm{C})$ using the $\mathrm{I}^{2} \mathrm{C}$ protocol. Compared to RTDs and thermopiles, both used extensively in the literature as temperature sensing elements in flow sensors, the digital temperature sensors have two advantages: firstly, the benefit of reduced noise since the measurement is converted into digital signal within the same chip. Secondly, it simplifies substantially the rest of the circuit design since there is no need for extra amplifiers or Wheatstone bridge. The sensor board is then mounted in a customized 3D printed housing box (Fig. 4a) made of PLA. It is designed to fit the sensor board perfectly, leaving only the sensing area exposed to the flow while providing access to the back of the board for the connections. The housing creates a cylindrical flow channel of $20 \mathrm{~mm}$ diameter and $96 \mathrm{~mm}$ of length.

\section{B. Auxiliary Board and Micro-controller}

An auxiliary (aux) PCB has also been designed to get the measurements from the temperature sensors and supply the heater with power. As Fig. $4 \mathrm{~b}$ depicts, the aux board is directly connected to the sensor board. As Fig. 3 shows, the aux board carries a demultiplexer (demux) and the analog switches, necessary for the communication between the $\mu \mathrm{C}$ and the temperature sensors. The reason is that all the digital temperature sensors have the same address, while the $\mu \mathrm{C}$ has only one data line (SDA) for the $\mathrm{I}^{2} \mathrm{C}$ protocol. Therefore, analog switches are used in order to connect the SDA from the $\mu \mathrm{C}$ to the right sensor every time when this is necessary. A 3-to-6 demux is used in order to reduce the number of connections to the $\mu \mathrm{C}$, thus, only three select signals are needed from the $\mu \mathrm{C}$ to control the six switches. The clock line (SCL) can be shared among the sensors without causing any issue. Also, the aux board has a pnp transistor, with its emitter connected to $5 \mathrm{~V}$

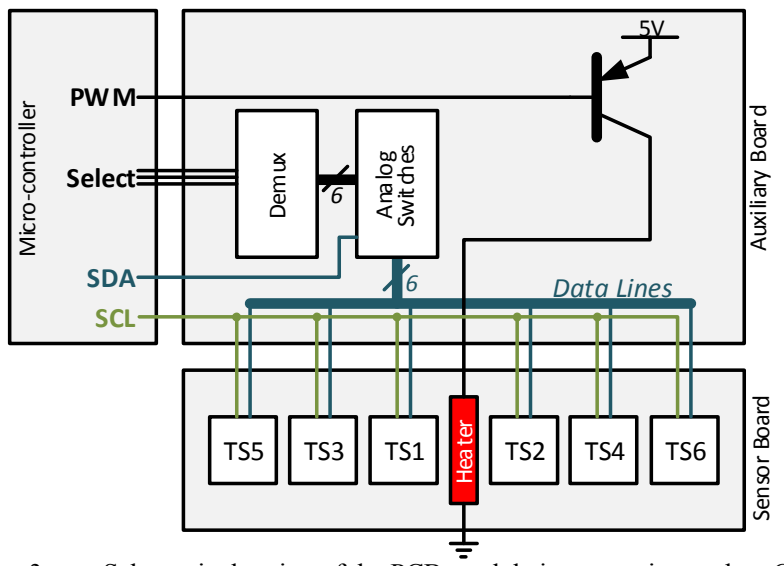

Fig. 3: Schematic drawing of the PCBs and their connection to the $\mu \mathrm{C}$.
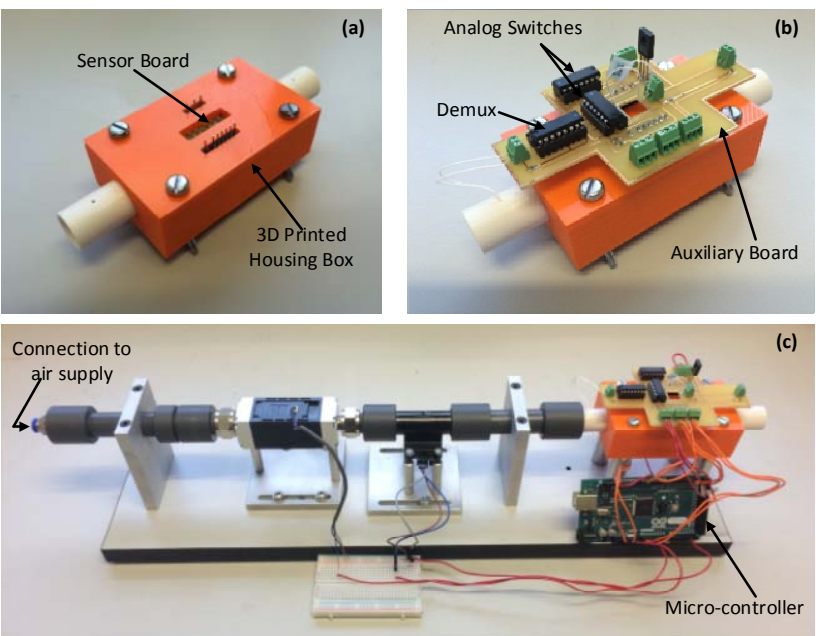

Fig. 4: Photos of the experiment's parts.

external supply, which is used to drive the heater. The power at the heater is regulated with the application of a PWM signal to the transistor's base coming from the $\mu \mathrm{C}$. Such a topology is preferred since the $\mu \mathrm{C}$ is not capable of providing the heater with the necessary power. For the experiments presented here, an Arduino ${ }^{\circledR}$ is used as a $\mu \mathrm{C}$ and the frequency of the PWM is at $490 \mathrm{~Hz}$. To acquire the temperature sensors' measurements, the $\mu \mathrm{C}$ asks all the IC sensors simultaneously to get a single measurement, hence, all the sensors start measuring at the exact same time. The typical time a sensor needs to get a single measurement is $66 \mathrm{~ms}$ and its last reading is stored within the IC chip. Then, the $\mu \mathrm{C}$ asks each sensor, one after the other, to send their readings. The whole process of measuring and sending the readings to the $\mu \mathrm{C}$ lasts $200 \mathrm{~ms}$, hence, the sampling frequency is set at $5 \mathrm{~Hz}$. This frequency is adequate for the majority of industrial and medical monitoring processes where only slow flow changes occur. The duty cycle of the PWM was set to $50 / 255$, unless otherwise stated, which corresponds to an analog voltage of $0.98 \mathrm{~V}$ at the base of the transistor. Subsequently, the current at the heater was measured at $0.383 \mathrm{~A}$, and the power at the heater at $1.47 \mathrm{~W}$. Note that the convective heat energy leaving the heater is directly related to the velocity of the fluid, hence, the power level supplied to the heater of a calorimetric sensor must increase in order to still be able to create a temperature gradient in high fluid velocities. 
The housing with the boards is then mounted in a setup of pipes, as Fig. 4c depicts, which facilitates the connection to fluid supply. The fluid used for the experiments is air and a Mass Flow Controller (MFC) is used to control the flow. Using the MFC, the user has the ability to determine the flow rate, as well as, to create different flow patterns and flow tests necessary for the experiments. The MFC used for this work can control and create flows up to 50 standard liters per minute (slm).

\section{EXPERIMENTAL RESULTS}

\section{A. Calibration}

Ideally, the temperature measurements between two symmetrical temperature sensors placed at the upstream, $T_{d}^{u p}$, and downstream, $T_{d}^{\text {down}}$, and at distance $d$, must be the equal when there is no flow, $\Delta T=T_{d}^{\text {down }}-T_{d}^{u p}=0$. However, this is not the case due to two main reasons: measurement error of the sensors and, the fact that their distances from the heater cannot be practically equal. Therefore, the offset of each pair is determined and removed. Before performing the experiments, the heater is on for 5 minutes without any flow through the air channel, providing more than enough time for the system to warm up. The average value of $\Delta T$ of the last minute for a measuring pair is calculated and removed as an offset from the measurements. As it was expected, the offset is larger for a pair closer to the heater than a pair far from it, since the ratio of misplacement, $\varepsilon_{\mathrm{d}}$, over the heater-to-temperature sensor distance, $d$, becomes smaller as $d$ increases.

\section{B. Symmetrical Measurements}

Fig. 5a shows the output of the flow sensor using symmetrically located sensing elements. After a certain flow rate, the output increases quickly for low velocities until it starts reaching a maximum, and then slowly decreasing almost linearly. Apparently, such a topology and mode of operation are quite limiting when the application requires accurate measurements of high fluid velocities. For the current test, the absolute gradient of the plots for flows between 25 to 50 liters is $0.062,0.044$ and 0.053 , for the pairs TS2-TS1, TS4-TS3 and TS6-TS5, respectively. Considering the resolution of the temperature sensors used, the flow resolution ideally would be at $0.16 \mathrm{slm}, 0.23 \mathrm{slm}$ and $0.19 \mathrm{slm}$, respectively. It must also be noted that the lowest resolution occurs for the pair closest to the heater.

\section{Asymmetrical Measurements}

Fig. 5b depicts the temperature difference between the sensor closest to the heater at the downstream (TS2) and sensor at the longest distance at the upstream (TS5), as well as, against the one in the middle (TS3), for the exact same measurement setup mentioned in the previous paragraph. Bear in mind that an offset is expected to occur due to the asymmetry and it is calculated and removed in the same way as in the symmetrical measurements. Firstly, note that the maximum output occurs for a lower flow rate than that of the symmetrical topology, noticeably increasing the flow range of linear output for the high fluid velocities. Most importantly, the gradient of the output for that range is now approximately 0.26 and 0.18 for the pairs TS2TS5 and TS2-TS3, respectively, ideally offering a flow
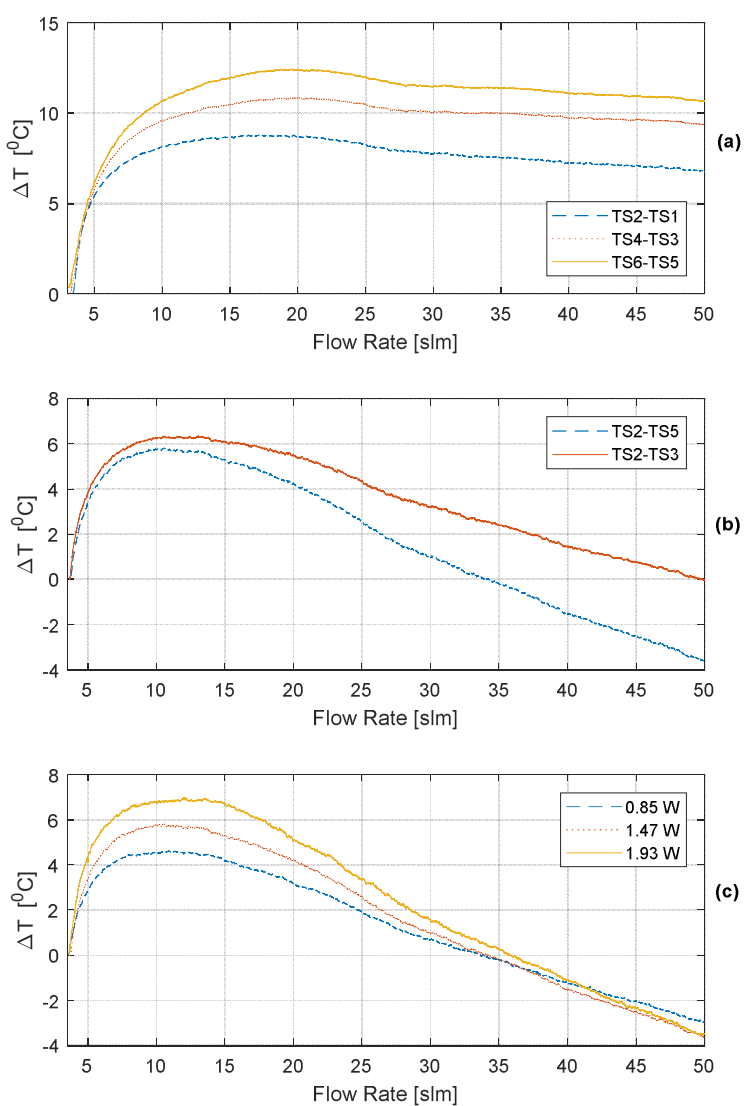

Fig. 5: Calorimetric flow sensor's output in CP using (a) symmetrical temperature sensors of different distances, (b) asymmetrical to the heater temperature sensors, and (c) the same asymmetric pair for different power.

resolution of $0.038 \mathrm{slm}$ and $0.056 \mathrm{slm}$, respectively again. This is a six fold improvement in resolution for the same power.

\section{Effect of Power}

This work also investigates the effect of different power applied to the heater on flow resolution for asymmetrically located sensing elements. Fig. 5c shows the flow sensor's output using the TS2-TS5 pair as measuring sensors, for two more power consumptions, one lower at $0.85 \mathrm{~W}$, and one higher at $1.93 \mathrm{~W}$, defined by different PWM duty cycles. It can be extracted from the data that the flow resolution is $0.048 \mathrm{slm}$, $0.038 \mathrm{slm}$ and $0.034 \mathrm{slm}$ for the $0.85 \mathrm{~W}, 1.47 \mathrm{~W}$ and $1.93 \mathrm{~W}$, respectively. It is proven that the trend for the flow resolution, when the power increases, is to also increase. However, it must be noted that the turn-over point moves to the right side with the increase of the power reducing the available flow range.

\section{DISCUSSION}

$\mathrm{CP}$ is commonly chosen due to its reliability and simple implementation, most of the times for low flow velocities and usually avoided for high flow rates due to its slowly changing output after the turn-over velocity. This work provides experimental evidence that asymmetrical locations of sensing elements can be used to practically solve that problem by increasing the sensitivity of the sensor for the same setup conditions while increasing that flow range by moving the turnover point to a lower velocity. 


\section{REFERENCES}

[1] Z. Yi, M. Qin, and Q. A. Huang, "Experiment of the MEMS Wind Sensor Based on Temperature-Balanced Mode," IEEE Sens. J., vol. 17, no. 8, pp. 2316-2317, 2017.

[2] M. Ahmed, W. Xu, S. Mohamad, M. Duan, Y. Lee, and A. Bermak, "Integrated CMOS-MEMS Flow Sensor With High Sensitivity and Large Flow Range,” IEEE Sens. J., vol. 17, no. 8, pp. 2318-2319, 2017.

[3] F. Hedrich, K. Kliche, M. Storz, S. Billat, M. Ashauer, and R. Zengerle, "Thermal flow sensors for MEMS spirometric devices," in Sensors and Actuators, A: Physical, 2010, vol. 162, no. 2, pp. 373378.

[4] Y. Zhu, B. Chen, M. Qin, and Q. A. Huang, "2-D Micromachined Thermal Wind Sensors - A Review," IEEE J. Internet Things, vol. 1, no. 3, pp. 216-232, 2014.

[5] J. T. W. Kuo, L. Yu, and E. Meng, "Micromachined thermal flow sensors-A review," Micromachines, vol. 3, pp. 550-573, 2012.

[6] K. Imin, A. Kumar, and J. Binder, "Smart MEMS Flow Sensor: Theoretical Analysis and Experimental Characterization," IEEE Sens. J., vol. 7, no. 5, pp. 713-722, 2007.

[7] P. Enoksson, G. Stemme, and E. Stemme, "A silicon resonant sensor structure for coriolis mass-flow measurements," IEEE J. Microelectromechanical Syst., vol. 6, no. 2, pp. 119-125, 1997.

[8] O. Berberig, K. Nottmeyer, J. Mizuno, Y. Kanai, and T. Kobayashi,
"The Prandtl micro flow sensor (PMFS): a novel silicon diaphragm capacitive sensor for flow-velocity measurement," Sensors Actuators, A Phys., vol. 66, no. 1-3, pp. 93-98, 1998.

[9] B. W. van Oudheusden, "Silicon thermal flow sensors," Sensors Actuators, A Phys., vol. 30, no. 1-2, pp. 5-26, 1992.

[10] T. S. J. Lammerink, N. R. Tas, M. Elwenspoek, and J. H. J. Fluitman, "Micro-liquid flow sensor," Sensors Actuators, A Phys., vol. 37-38, pp. 45-50, 1993.

[11] N. T. Nguyen and W. Dötzel, "Asymmetrical locations of heaters and sensors relative to each other using heater arrays: a novel method for designing multi-range electrocaloric mass-flow sensors," Sensors Actuators, A Phys., vol. 62, pp. 506-512, 1997.

[12] V. Kitsos, S. West, A. Demosthenous, and X. Liu, "Design considerations and optimization of calorimetric flow sensor for respiratory monitoring," in IEEE Biomedical Circuits and Systems Conference (BioCAS), 2016, no. 2, pp. 26-29.

[13] N. Sabaté, J. Santander, L. Fonseca, I. Gràcia, and C. Cané, "Multirange silicon micromachined flow sensor," Sensors Actuators, A Phys., vol. 110, pp. 282-288, 2004.

[14] A. Petropoulos and G. Kaltsas, "Study and evaluation of a PCBMEMS liquid microflow sensor," Sensors, vol. 10, no. 10, pp. 89819001, 2010. 